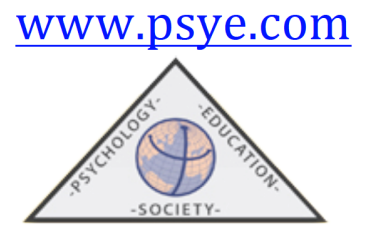

C Psychology, Society, \& Education, 2019. Vol. 11(2), pp. 193-204 ISSN 2171-2085 (print) / ISSN 1989-709X (online)

Doi 10.25115/psye.v10i1.2024

\title{
Análisis factorial, fiabilidad y validez de la escala de autoeficacia general (EAG) en estudiantes ecuatorianos
}

\author{
Rodrigo MORETA-HERRERA ${ }^{1}$, Mariela LARA-SALAZAR ${ }^{2}$, \\ Pamela CAMACHO-BONILLA ${ }^{1}$ y Sandy SÁNCHEZ-GUEVERA ${ }^{1}$
}

\author{
${ }^{1}$ Pontificia Universidad Católica del Ecuador - Ambato \\ ${ }^{2}$ Universidad Técnica de Ambato
}

(Recibido, el 28 de Junio de 2018; Aceptado, el 26 de Noviembre de 2018)

\begin{abstract}
RESUMEN: Objetivos: Confirmar la estructura factorial unidimensional de la Escala de Autoeficacia General (EAG), verificar su fiabilidad en adolescentes y jóvenes ecuatorianos, así como la validez. Método: La investigación es cuantitativa e instrumental, que analiza la estructura factorial, la fiabilidad y la validez de la EAG. Participantes: 742 adolescentes y jóvenes adultos, estudiantes de centros educativos de las ciudades de Ambato y Pelileo en Ecuador. Resultados: A nivel factorial la EAG con un solo factor explica el 52,1\% de la varianza con un ajuste óptimo $X^{2}=82,06 ; \mathrm{gl}=28 ; p<, 001 ; X^{2} / \mathrm{gl}=$ 2,93; GFI= ,96; NFI= ,93; CFI= ,96; SRMR= ,04; RMSEA= ,06; IC 90\% [,05 - ,08]. Además, es fiable para jóvenes con $\alpha=, 896$ y adolescentes con $\alpha=0,876$ y válida con pruebas de procrastinación. Conclusiones: La EAG se presenta como una prueba estandarizada para la población ecuatoriana de adolescentes y jóvenes, además de fiable y válida para el uso en la investigación.
\end{abstract}

Palabras clave: autoeficacia general, estructura factorial, fiabilidad, validez

Factor analysis, reliability and validity of the general self-efficacy scale (GSE) in ecuadorian students

ABSTRACT: Aims: To confirm the one-dimensional factor structure of the General Self-Efficacy Scale (EAG), verify its reliability in ecuadorian adolescents and young people, as well as its validity. Method: The research is quantitative and instrumental, which analyzes the factor structure, reliability and validity of EAG. Sample: 742 teenagers and young adults, students from educational centers in Ambato and Pelileo cities from Ecuador. Results: At the factorial level, the EAG with a single factor explains $52.1 \%$ of the variance with an optimal adjustment $X^{2}=82,06 ; \mathrm{df}=28 ; p<, 001 ; X^{2} / \mathrm{df}=2,93$; $\mathrm{GFI}=, 96 ; \mathrm{NFI}=, 93 ; \mathrm{CFI}=, 96$; $\mathrm{SRMR}=, 04 ; \mathrm{RMSEA}=, 06$; IC $90 \%[, 05-, 08]$. It is also reliable for young $\alpha=, 896$ and teenagers $\alpha=0,876$ and valid with procrastination tests. Conclusions: The EAG is presented like a standardized test for teenagers and young ecuadorian people, in addition to being reliable and valid for use in research.

Keywords: general self-efficacy, factor structure, reliability, validity

Correspondencia: E-mail: rmoreta@pucesa.edu.ec 


\section{Introducción}

La autoeficacia general comprende la creencia sobre la capacidad individual para manejar una amplia gama de situaciones (Suárez, García, \& Moreno, 2000). Este es un concepto que se remonta a los estudios de Bandura (1992), en el que se hace referencia a la 'expectativa de autoeficacia', y comprende el sentimiento de confianza en las propias capacidades resolutivas o de afrontamiento del individuo ante la cotidianidad. Además, evalúa también la competencia para manejarse eficazmente en una variedad de situaciones tensas (Baessler \& Schwarzer, 1996). Y permite la reducción del estrés y facilita la culminación de un objetivo (Merino Tejedor \& Lucas Mangas, 2016), como pueden ser los objetivos académicos.

El estudio de la autoeficacia en la educación, entrama una notoria importancia para entender el contexto de las situaciones y las conductas académicas (Blanco Vega, Ornelas Contreras, Rueda Villen \& Martínez Marín, 2013), en el que interviene la sensibilidad de la población (niños, adolescentes, jóvenes) y el impacto de la educación en el tejido social. Diversos estudios preliminares revelan que la autoeficacia se la analiza para conocer la relación con la resolución de problemas y la comunicación científica (Flores, Mayorga-Vega, Blanco \& Blanco, 2014); el impacto en el rendimiento académico (Gutiérrez, Corrás, Novo \& Fariña, 2017) e incluso las diferencias por género (Aguirre, Blanco, Rodríguez-Villalobos \& Ornelas, 2015), entre otros.

La autoeficacia general, se evalúa con la Escala de Autoeficacia General (EAG, Jerusalem \& Schwarzer (1992); Schwarzer (1993), como una herramienta diseñada para medir las capacidades de los individuos en el manejo y resolución de situaciones cotidianas estresantes. Es una batería de frecuente predilección para su uso, por lo que cuenta con varias traducciones, adaptaciones idiomáticas y validaciones ajustadas a su estructura clásica unidimensional.

Entre las distintas traducciones y adaptaciones, se encuentren las realizadas en muestras alemanes, costarricenses y chinas con fiabilidad $(\alpha)$ fluctuante entre ,81 (Costa Rica) y ,91 (China); que con un solo factor explican la varianza en $41 \%$ para alemanes, $39 \%$ en costarricenses y $55 \%$ en chinos y con convergencia en pruebas de ansiedad, depresión y optimismo (Schwarzer et al., 1997). También en muestra suiza con pacientes con lesiones medulares que confirman la estructura unifactorial y con confiabilidad de $\alpha=, 92$ (Peter, Cieza $\&$ Geyh, 2014). Mientras que en traducciones en idioma español se muestran estudios en España en adolescentes explicando el $51,9 \%$ de la varianza y además es fiable con $\alpha=, 89$ así como estable en el tiempo (Espada et al., 2012).

Ya en el contexto latinoamericano, hay estudios en el Perú con adolescentes escolarizados, en el que se señala la presencia de una estructura unifactorial que explica el $32 \%$ de la varianza, que además converge con escalas de Calidad de Vida $(r=, 31 ; p<, 001)$ y con una confiabilidad de $\alpha=, 75$ (Grimaldo Muchotrigo, 2005). En muestras combinadas de México y España en universitarios se confirmó la estructura unidimensional con 46,4\% (México) y $43,1 \%$ (España) de explicación de la varianza, además de fiable con $\alpha=, 79$ y $\alpha=, 77$ respectivamente y que converge con Automonitoreo y Control de la Acción (Padilla et al., 2006). En Chile, también se analizó en población general de 15 a 65 años, en el que se encontró una fiabilidad de $\alpha=, 84$ con validez de constructo adecuado y convergente con pruebas de autoestima, aunque sin un análisis factorial (Cid, Orellana \& Barriga, 2010).

También se encontraron casos, en los que la tendencia unidimensional de la prueba no fue encontrada, como por ejemplo Argentina con población general, en el que los hallazgos, 
mostraron dos factores con una explicación del $44 \%$ de la varianza; con fiabilidad de ,76 y convergente con optimismo, locus de control y afrontamiento; aunque sin un análisis factorial confirmatorio (Brenlla, Aranguren, Rossaro, Vázquez, 2010).

La revisión de los estudios con respecto a la medición de la EAG, muestran como tendencia general, que la EAG es una prueba fiable y con una estructura factorial unidimensional (aunque no es la única). Sin embargo, muchos de estos análisis revelan limitaciones en la verificación de la estructuración teórica, pues generalmente muestran análisis a nivel Exploratorio (AFE), sin que se complementen con los de tipo factorial confirmatorio (AFC) u otras pruebas. En el caso del Ecuador, se debe mencionar que la realización de adaptación y validación de pruebas es pobre. Destacan ciertos estudios como los de Peña Contreras y otros (2017) y Moreta-Herrera, Duran-Rodriguez \& Gaibor-Gonzalez (2018), pero para la autoeficacia es nula.

Aun así, estos estudios realizados en varios contextos y poblaciones al identificar la unidimensionalidad de la prueba para medir la autoeficacia general, ha llevado a teorizar en ciertos autores la propiedad de 'transculturalidad' de la prueba que otorga confianza evaluatoria de forma invariante a los elementos culturales específicos de las distintas poblaciones (Scholz, Doña, Sud \& Schwarzer, 2002). Aunque, hay también, posturas detractoras consideran a esta hipótesis como incorrecta, y que puede fenómeno puede ser explicado debido a los sesgos metodológicos que se derivan de las pruebas estadísticas utilizadas y el uso inadecuado de las estimaciones (Damásio et al., 2016).

Desde este punto de vista, consideramos que un proceso de análisis de las propiedades psicométricas del EAG en poblaciones de nacionalidades distintas a los estudios revisados, pueden contribuir a la estimación de la hipótesis de transculturalidad del EAG como es el caso de la población ecuatoriana en el que no se tienen estudios referenciales y de homologación del constructo.

Así mismo, llama la atención que entre los mecanismos de validación de la EAG la validez de convergencia, se la usa con pruebas no ligadas específicamente a la educación como los de salud, calidad de vida, automonitoreo, autoestima y otros aun cuando en su mayoría las muestras corresponden a adolescentes y jóvenes escolarizados. Esta observación nos permite estimar que el control de la validez requiere del uso de pruebas ligadas a la educación y las conductas académicas. Esto por la particularidad de la población estudiante, la vulnerabilidad y la exposición a riesgos existentes (Moreta, Reyes, Mayorga \& León-Tamayo, 2017; MoretaHerrera, Durán-Rodríguez \& Gaibor-González, 2018; Moreta-Herrera et al., 2018) y por el impacto que tiene la autoeficacia en la salud mental (Chau \& Vilela, 2017) para el desarrollo adecuado del aprendizaje.

\section{Objetivos e hipótesis}

Para el presente estudio, se espera como objetivos de trabajo: a) confirmar la estructura factorial unidimensional de la EAG en estudiantes ecuatorianos, como lo preveen estudios anteriores en otras poblaciones; b) corroborar la hipótesis de universalidad del constructo, predicha por Scholz, Doña, Sud \& Schwarzer (2002); c) conocer la fiabilidad de la EAG, pues se estima que esta es elevada; y, d) Ratificar la validez de constructo de la EAG y la convergencia con pruebas de conducta procrastinadora en estudiantes. 


\section{Metodología}

\section{Diseño}

El trabajo comprende un estudio de tipo cuantitativo de carácter instrumental (Montero \& León, 2007) en el que se busca identificar la estructura factorial y confirmarla, además de determinar la fiabilidad y la validez convergente de la EAG.

\section{Participantes}

La muestra estuvo constituida por 742 participantes agrupados en dos segmentos. El primero por 400 estudiantes universitarios (62,5\% mujeres y 27,5\% hombres) con edades entre 18 a 25 años $(M=21,1$ años; $D T=1,95)$. El 97,8\% se definen como mestizos(as) y los demás como indígenas $(1,2 \%)$ y blancos $(1 \%)$. Además, el $86 \%$ se encuentran domiciliados en la zona urbana y el $14 \%$ en la rural. El $24,8 \%$ se encuentran en situación de vulnerabilidad económica. Son estudiantes de una universidad privada con el $62,5 \%$ y una universidad pública que representa el 27,5\%. Vinculados(as) a las carreras de: a) Psicología (37,5\%), Laboratorio Clínico (25\%), Jurisprudencia y Enfermería con 12,5\% cada una; y Contabilidad y Administración (12,5\%). El $13,3 \%$ combinan sus estudios con actividades laborales y el $2 \%$ repiten nivel.

El segundo grupo son 342 adolescentes de bachillerato (49,1\% hombres y 50,9\% mujeres) con edades entre 14 a 20 años $(M=16,18 ; D T=1,1)$. El 93,9\% se definen como mestizos y el $6,1 \%$ como indígenas. El $64 \%$ se domicilian en la zona urbana y el $36 \%$ en la rural. Además, el 20,2\% se encuentra en vulnerabilidad socioeconómica. Los participantes son estudiantes de dos colegios públicos, cursantes del primer $(28,4 \%)$, segundo $(33,6 \%)$ y tercer (38\%) año de bachillerato. Además, un 9,1\% señala que repite curso, mientras que el $7 \%$ combina sus estudios con actividades laborales.

La selección de los participantes se hizo a través de un muestreo no probabilístico con criterios de inclusión: a) Ser estudiante legalmente matriculado; b) Tener asistencia regular; c) Firmar carta de consentimiento (tutor en el caso de los menores de edad); y d) Poseer estado de salud físico y mental aceptable para someterse al proceso de evaluación psicológica.

\section{Instrumentos}

Escala de Autoeficacia General (EAG, Schwarzer, 1993), en la versión de Baessler y Schwarzer (1996). Consta de 10 ítems con respuestas en escalas de tipo Likert de 5 puntos entre 1 (totalmente en desacuerdo) a 5 (totalmente de acuerdo). Los puntajes entre 27 a 38 puntos muestran una media de autoeficacia general. Esta es fiable con valores de $\alpha=, 87$ para la versión española.

Escala de Procrastinación (Busko, 1998) en la versión peruana de Álvarez (2010). Cuenta con dos pruebas: a) La Escala de Procrastinación General (EPG) que consta de 12 ítems; y b) La Escala de Procrastinación Académica (EPA) la cual consta de 16. Las respuestas a los ítems se registran en una escala likert de cinco puntos de 1 (nunca) a 5 (siempre). Los puntajes elevados señalan una presencia significativa de procrastinación general y académica. Se puede mencionar que en la versión peruana se encontraron valores de fiabilidad altos de ,87 y,80. 


\section{Procedimiento metodológico}

Posterior a la autorización de los centros educativos para las aplicaciones de la batería, los participantes fueron informados del proyecto y los objetivos del caso. Las evaluaciones fueron aplicadas de manera grupal en los salones de clase de sus respectivos centros de estudio. Los participantes firmaron la carta de consentimiento para la evaluación en el caso de la muestra de universitarios, mientras que en el caso de los adolescentes lo llenaron sus tutores legales. Una vez concluido las evaluaciones, éstas fueron revisadas y depuradas para la tabulación y posterior análisis estadístico. Además, el reporte final con los resultados fue enviado previamente a la Comisión de Publicaciones de la PUCE-Ambato que ejerce las funciones de evaluación ética de la propuesta para la posterior publicación. La gestión informática de los resultados se realizó con los programas informativo SPSS versión 21 (IBM Corp., 2012) que comprenden los análisis preliminares, el AFE, la fiabilidad y validez; y, el programa AMOS versión 24 (Arbuckle, 2014) para el AFC.

\section{Resultados}

\section{Análisis preliminar de los ítems}

Como se observa en la tabla 1, los 10 ítems del EAG muestran medias que oscilaron entre $M=3,75$ en el ítem cuatro y $M=4,21$ en el ítem dos, señalando su homogeneidad y la tendencia a marcar puntuaciones elevadas. En los análisis de comprobación del supuesto de normalidad multivariada, a través de la estimación de los índices de la asimetría y curtosis, se confirma el cumplimiento del supuesto cuando los puntajes de los índices se encuentran dentro del umbral $\pm 1,5$ (Cuadras, 2016). En los análisis se observa en la curtosis del ítem dos que este se encuentra fuera de dicho parámetro, por lo que se puede inferir la ausencia de normalidad multivariada. $\mathrm{Y}$ en referencia al diagnóstico de multicolinealidad, el cual se considera existente cuando las puntuaciones del análisis de correlación ítem-test corregido $\left(r_{(i-t) c}\right)$ son superiores a ,9 (Kline, 2011), los resultados descartan la presencia de redundancia entre los ítems o una excesiva baja correlación por lo que se muestra innecesario la eliminación de ítems para el análisis factorial exploratorio (AFE).

Tabla 1. Análisis preliminar de los items

\begin{tabular}{lccccc}
\hline Ítems & $\boldsymbol{M}$ & $\boldsymbol{\sigma}$ & Asimetría & Curtosis & $\boldsymbol{r}_{(i-t c)}$ \\
\hline Ítem 01 & 3,84 & 0,93 & $-0,66$ & 0,26 &, 56 \\
Ítem 02 & 4,21 & 0,77 & $-1,06$ & 1,78 &, 63 \\
Ítem 03 & 3,99 & 0,87 & $-0,84$ & 0,72 &, 55 \\
Ítem 04 & 3,94 & 0,81 & $-0,74$ & 0,94 &, 62 \\
Ítem 05 & 3,96 & 0,86 & $-0,76$ & 0,59 &, 67 \\
Ítem 06 & 3,75 & 0,93 & $-0,64$ & 0,35 &, 62 \\
Ítem 07 & 3,79 & 0,87 & $-0,47$ & 0,09 &, 75 \\
Ítem 08 & 4,02 & 0,82 & $-0,76$ & 0,47 &, 70 \\
Ítem 09 & 3,81 & 0,88 & $-0,54$ & 0,03 &, 63 \\
Ítem 10 & 3,90 & 0,88 & $-0,60$ & 0,07 &, 70 \\
\hline
\end{tabular}

Nota: $r(i-t c)$ : Correlación item-test corregida 


\section{Análisis factorial}

Análisis exploratorio. Se tomó en cuenta el tamaño de la muestra, que fue de 400 estudiantes universitarios, autores como Ferrando y Anguiano-Carrasco (2010) sugieren un número mayor a 200, mientras que Tabachnick y Fidell (2007) consideran al menos 300. Las pruebas de adecuación factorial muestran que el EAG presenta valores de $\mathrm{KMO}=, 92$ que se considera como adecuado cuando se basan en matrices Pearson (Domínguez Lara, 2014); además, es óptima para la evaluación de la aplicabilidad del AFE, como arrojan los resultados de la prueba de Esfericidad de Bartlett $X^{2}=1856,9 ; p<, 001$.

El método extracción de componentes principales (MCP) estimó la presencia de un solo factor en la EAG que logra explicar el 52,1\% de los cambios en la varianza. Esto señala estimación adecuada de la prueba tal como se encuentra estructurada en la tabla dos. En el reporte de comunalidades $\left(\mathrm{h}^{2}\right)$ se encontró valores que fluctuaron entre ,404 (ítem 3) y ,660 (ítem 1); de estos datos se desprenden la homogeneidad de las comunalidades que no arrojan valores extremos y que indiquen la ausencia de multicolinealidad o redundancia entre los ítems.

Tabla 2. Análisis Factorial Exploratorio con el Método de Componentes Principales

\begin{tabular}{rlrc}
\hline Ítems & & $\boldsymbol{h}^{2}$ & $\boldsymbol{F ~ I ~}$ \\
\hline 07 & Venga lo que venga, por lo general soy capaz de manejarlo &, 666 &, 816 \\
08 & Puedo resolver la mayoría de los problemas si me esfuerzo lo necesario &, 596 &, 772 \\
10 & $\begin{array}{l}\text { Al tener que hacer frente a un problema, generalmente se me ocurren varias } \\
\text { alternativas de cómo resolverlo }\end{array}$ &, 594 &, 770 \\
05 & Gracias a mis cualidades puedo superar situaciones imprevistas &, 557 &, 746 \\
09 & Si me encuentro en una situación difícil, generalmente se me ocurre qué debo hacer &, 510 &, 714 \\
06 & Cuando me encuentro en dificultades puedo permanecer tranquilo (a) porque cuento &, 496 &, 704 \\
02 & con las habilidades necesarias para manejar situaciones difíciles &, 495 &, 703 \\
04 & Tengo confianza en que podría manejar eficazmente acontecimientos inesperados &, 483 &, 695 \\
01 & Puedo encontrar la manera de obtener lo que quiero aunque alguien se me oponga &, 413 &, 643 \\
03 & Me es fácil persistir en lo que me he propuesto hasta llegar a alcanzar mis metas &, 404 &, 636 \\
\hline
\end{tabular}

Varianza: $52,1 \%$

$\boldsymbol{K M O}=0,916 ;$ Bartlett: $X^{2}=1856,90 ; p<0,001$

\section{Análisis confirmatorio}

Es importante mencionar, las consideraciones que se tomaron en cuenta para garantizar la generalización de la prueba en diversos segmentos poblacionales como: a) El tamaño de la muestra para un adecuado AFC que debió ser mayor a 200 participantes (Brown, 2015); y b) la confirmación de la prueba con una muestra de adolescentes que son distintos a los del AFE; a fin de mejorar la 'no-representatividad' de las muestras (Ferrando \& Anguiano-Carrasco, 2010).

El AFC con estimación de Máxima Verosimilitud, muestra que el modelo de ajuste unidimensional del EAG es el más óptimo como se hipotetizó previamente en el AFE y como se puede observar en la figura 1 . 
En las pruebas de ajuste robusto hay significación estadística, lo cual indicaría que el ajuste no es el adecuado, pero dado el tamaño de la muestra para el AFC, se consideraría difícil la ausencia de significancia estadística, es por lo tanto, que se requirió de la Razón Chi cuadrado normado $\left(X^{2} / d f\right)$ para justificar o no la calidad del ajuste. Para el presente caso los resultados fueron de $X^{2} / d f=2,93$ valor equivalente a ajuste adecuado (Byrne, 2006). Por otra parte, los modelos de ajuste muestran ser óptimos por los valores encontrados en el GFI, NFI, CFI, SRMR y RMSEA (Abad, Olea, Ponsoda \& García, 2011), todos ellos dentro de los parámetros de ajuste establecidos previamente.

Los parámetros de ajuste robusto y normado son $X^{2}=82,06 ; \mathrm{df}=28 ; p<, 001 \mathrm{y} X^{2} / d f=$ 2,93; mientras que los índices de bondad son $\mathrm{GFI}=, 96 ; \mathrm{NFI}=, 93 ; \mathrm{CFI}=, 96 ; \mathrm{SRMR}=, 04$; RMSEA= ,06 IC 90\% [,05 - ,08]. Con todo esto, podemos mencionar que el EAG es una prueba unidimensional con 10 ítems de elevado ajuste y cuyas cargas factoriales oscilan entre ,55 y ,71; las mismas que se consideran homogéneas entre sí.

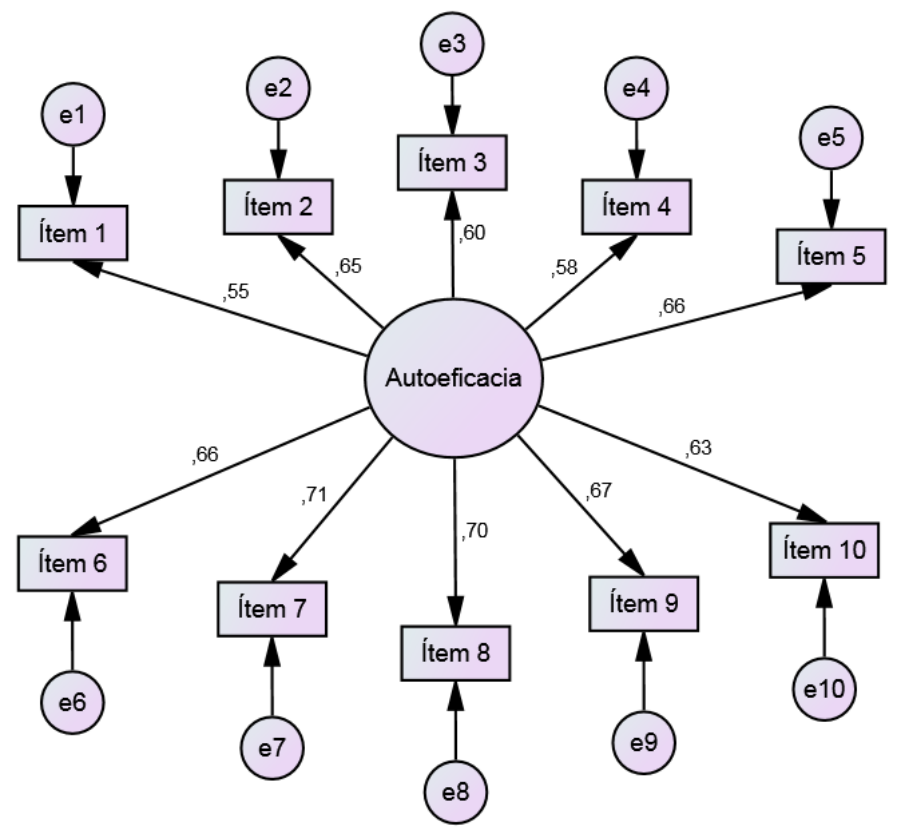

$X^{2}=82,06 ; d f=28 ; p<0,001 ; X^{2} / d f=2,93 ; G F I=0,96 ; N F I: 0,93 ; C F I: 0,96 ; R M S E A=0,06[0,05-0,08]$

Estimación: Máxima Verosimilitud

Figura 1: Análisis factorial confirmatorio de la Escala de Autoeficacia General. La elipse representa al factor y los rectángulos representan los diferentes items.

Las varianzas residuales se muestran en los círculos pequeños

\section{Análisis de fiabilidad y validez.}

El cálculo de la fiabilidad con el coeficiente de Cronbach muestra que es alta y consistente, tanto para la muestra de adultos universitarios $\alpha=0,896$ como de adolescentes $\alpha=$ 0,876; el mismo que confirma la adecuación de la fiabilidad con la segunda prueba basada en las mitades de dos mitades de Guttman que es 0,833 para el primero y 0,854 para el segundo. Aunque se debe anotar como se visualiza en la tabla tres, la presencia de ligeras diferencias en 
el cálculo de la misma, esto probablemente por la ausencia de normalidad multivariada o el método de cálculo seleccionado.

Tabla 3. Análisis de fiabilidad de la EAG

\begin{tabular}{lll}
\hline Muestras & Cronbach & Guttman \\
\hline Adultos & 0,896 & 0,833 \\
Adolescentes & 0,876 & 0,854 \\
\hline
\end{tabular}

Nota: $E A G=10$ items

Con respecto a la validez de constructo, en la tabla cuatro, se puede observar que los pares de correspondencias de los ítems, muestran una marcada homogeneidad entre las mismas. De manera general éstas se enmarcan como correlaciones bajas y moderadas positivas. Los coeficientes oscilaron entre $r=, 301$ en el par de los ítems 9-3 y $r=, 647$ en el par 9-10. A esto, también hay que agregar que los análisis de correlación ítem-test del análisis preliminar de la tabla 1 señalan resultados moderados y alto con valores entre $r=, 553$ en el ítem $3 \mathrm{y} r=, 750$ en el ítem 7. En síntesis, se puede considerar que la EAG cuenta con una significativa validez de constructo.

Tabla 4. Análisis de validez de constructo de la EAG

\begin{tabular}{lllllllllll}
\hline & $\mathbf{0 1}$ & $\mathbf{0 2}$ & $\mathbf{0 3}$ & $\mathbf{0 4}$ & $\mathbf{0 5}$ & $\mathbf{0 6}$ & $\mathbf{0 7}$ & $\mathbf{0 8}$ & $\mathbf{0 9}$ & $\mathbf{1 0}$ \\
\hline 01 & 1 & & & & & & & & & \\
02 &, $472^{* *}$ & 1 & & & & & & & & \\
03 &, $386^{* *}$ &, $533^{* *}$ & 1 & & & & & & & \\
04 &, $342^{* *}$ &, $445^{* *}$ &, $454^{* *}$ & 1 & & & & & & \\
05 &, $378^{* *}$ &, $453^{* *}$ &, $448^{* *}$ &, $618^{* *}$ & 1 & & & & & \\
06 &, $372^{* *}$ &, $373^{* *}$ &, $352^{* *}$ &, $482^{* *}$ &, $536^{* *}$ & 1 & & & & \\
07 &, $467^{* *}$ &, $448^{* *}$ &, $387^{* *}$ &, $492^{* *}$ &, $554^{* *}$ &, $572^{* *}$ & 1 & & & \\
08 &, $455^{* *}$ &, $534^{* *}$ &, $408^{* *}$ &, $421^{* *}$ &, $478^{* *}$ &, $478^{* *}$ &, $637^{* *}$ & 1 & & \\
09 &, $370^{* *}$ &, $347^{* *}$ &, $301^{* *}$ &, $396^{* *}$ &, $456^{* *}$ &, $429^{* *}$ &, $624^{* *}$ &, $529^{* *}$ & 1 & \\
10 &, $457^{* *}$ &, $500^{* *}$ &, $402^{* *}$ &, $384^{* *}$ &, $445^{* *}$ &, $474^{* *}$ &, $622^{* *}$ &, $572^{* *}$ &, $647^{* *}$ & 1 \\
\hline Nota: $* *$ & $p<0,01$ & & & & & & & & & \\
\hline
\end{tabular}

Por último, en la tabla 5 se observan los cálculos de la validez convergente con la prueba de Procrastinación (Busko, 1998; Álvarez, 2010). La prueba arroja convergencia negativa baja con los componentes General con $r=-, 29 ; p<, 01$ y Académica con $r=-, 32 ; p<, 01$ para los universitarios. Mientras que, en los adolescentes, solo converge con la Académica con $r=-, 31 ; p<, 01$. 
Tabla 5. Análisis validez de convergencia del EAG con la Procrastinación

\begin{tabular}{llc}
\hline \multirow{2}{*}{ Muestras } & \multicolumn{2}{c}{ Procrastinación } \\
\cline { 2 - 3 } & General & Académica \\
\hline Adultos & $-0,29 * *$ & $-0,32^{* *}$ \\
Adolescentes & $-0,05^{* *}$ & $-0,31^{* *}$ \\
\hline Nota: ${ }^{* *} p<0,01$ & &
\end{tabular}

\section{Discusión}

Se propusieron como objetivos del trabajo la confirmación de la EAG como una prueba unifactorial en población ecuatoriana, además de ser altamente fiable y válida como constructo y convergente con la procrastinación.

Desde el punto de vista de la normalidad multivariada, la evaluación con la EAG en la población de estudiantes adolescentes y universitarios mostró la no existencia del supuesto de normalidad multivariada. Sobre el análisis factorial, tanto a nivel exploratorio como confirmatorio se determinó que es una prueba unidimensional, como previamente fue hipotetizado. La explicación de la varianza $(52,1 \%)$, se considera como adecuada y con una mayor confianza de la prueba en nuestro contexto que en el de los estudios referenciales en otros países. Estos datos concuerdan con conclusiones similares de la unifactorialidad de la prueba en los reportes en alemanes, costarricenses y chinos (Schwarzer et al., 1997); suizos (Peter, Cieza \& Geyh, 2014); españoles (Baessler \& Schwarzer, 1996; Suárez, García \& Moreno, 2000; Espada et al. 2012), chilenos (Cid, Orellana \& Barriga, 2010), peruanos (Grimaldo Muchotrigo, 2005) y mexicanos (Padilla et al., 2006). Aunque no en argentinos, quienes reportaron dos factores, (Brenlla et al., 2010). Hay que considerar que el aporte del AFC de este trabajo, consolida las conclusiones previamente halladas.

Además, cabe destacar que la unidimensionalidad de la prueba se presenta, aún con las diferencias de contexto que componen los grupos de la muestra, ya que se probó el AFE en universitarios y el $\mathrm{AFC}$ en adolescentes de bachillerato. Esto precisamente se realizó para garantizar la generalidad de prueba considerando las opiniones de Ferrando y AnguianoCarrasco (2010). Este mecanismo de diseño del análisis factorial, es pionero en estudios similares y también en el Ecuador. Pero además nos permite concluir su utilidad para estudiantes a nivel de bachillerato y universitarios como prueba adecuada de la autoeficacia.

Dado que los análisis factoriales verificaron la unifactorialidad de la prueba en ecuatorianos, estos resultados encontrados nos permiten contribuir a la hipótesis de 'transculturalidad' del constructo de autoeficacia mencionado por Scholz et al. (2002). Esto viene dado previamente por la cantidad de estudios que arrojan resultados similares en más de 25 países con distintos tipos de muestras que incluyen a adolescentes, universitarios, población general y otros. Sin embargo, no se deben descuidar aspectos como el género y las diferencias culturales significativas o la traducción del test, ya que pueden influir en los resultados (Damásio et al., 2016).

Finalmente, se demuestra que la EAG es válida a nivel de constructo por la homogeneidad en los coeficientes de intercorrelaciones y de correlación ítem-test y también en 
términos de convergencia con la procrastinación académica por el tipo de muestra trabajado muy cercano al contexto educativo. Aunque se debe considerar que no hay estudios previos referenciales con este tipo de pruebas.

A modo de conclusión, la EAG es una prueba útil para la identificación de la autoeficacia y de preferencia en la aplicación para muestras de adolescentes de bachillerato y de jóvenes universitarios; aunque en otro tipo de muestras, su uso debe ser tomado con precaución. Las implicaciones de este estudio se enmarcan en el potencial de esta prueba para el análisis de la eficacia en aspectos como el educativo para la adquisición de competencias académica; también en el campo clínico como un estimador de la intervención psicológica o en campo laboral o social en el aspecto resolutivo de situaciones o circunstancias cotidianas.

\section{Limitaciones y nuevas líneas de investigación}

Entre las limitaciones de la prueba se debe estimar que se trabajó con adolescentes y jóvenes universitarios pertenecientes a las ciudades de Ambato y Pelileo en la provincia de Tungurahua, por lo que se requerirán nuevos estudios que integren a personas de otras zonas geográficas del país. Por otra parte, fueron incluidos solo estudiantes de bachillerato y universidad, razón por la que se requieren mayores análisis con muestras de otras poblaciones como la general, clínica, de tercera edad, en otros.

Desde el punto de vista metodológico se debe considerar la necesidad de indagar en la fiabilidad con otras pruebas alternativas como la estabilidad temporal y la validez discriminante para garantizar la aplicabilidad del test en la población ecuatoriana.

\section{Referencias}

Abad, F., Olea, J., Ponsoda, V., \& García, C. (2011). Medición en Ciencias Sociales y de la Salud. Madrid: Síntesis.

Aguirre, J. F., Blanco, J. R., Rodríguez-Villalobos, J. M., \& Ornelas, M. (2015). Autoeficacia General Percibida en Universitarios Mexicanos, Diferencias entre Hombres y Mujeres. Formación universitaria, 8(5), 97-102.

Álvarez, O. (2010). Procrastinación general y académica en una muestra de estudiantes de secundaria de Lima metropolitana. Persona, 13, 159-177.

Arbuckle, J. L. (2014). Amos (Version 23.0) [Computer Program]. Chicago: IBM SPSS.

Baessler, J., \& Schwarzer, R. (1996). Evaluación de la autoeficacia: Adaptación española de la escala de Autoeficacia General. Ansiedad y Estrés, 2, 1-8.

Bandura, A. (1992). Exercise of personal agency through the self-efficacy mechanism. En R. Schwarcer (Ed.), Self-efficacy: Thought control of action (págs. 3-38). Washinton, DC: Hemisphere.

Blanco Vega, H., Ornelas Contreras, M., Rueda Villen, M., \& Martínez Marín, M. (2013). Composición factorial de la escala de autoeficacia en conductas académicas en universitarios de ciencias sociales. Revista Mexicana de Psicología, 30(1), 79-88.

Boomsma, A., \& Hoogland, J. (2001). The robustness of LISREL modeling revisited. En R. Cudeck, S. Du Toit, \& D. Sörbom (Edits.), Structural equation modeling: present and future. A festschrift in honor of Karl Jöreskog (págs. 139-168). Chicago: Scientific Software International. 
Brenlla, M. E., Aranguren, M., Rossaro, M. F., \& Vázquez, N. (2010). Adaptación para Buenos Aires de la escala de autoeficacia general. Interdisciplinaria, 27(1), 77-94.

Brown, T. A. (2015). Confirmatory factor analysis for applied research (Vol. 2nd). New York: Guilford Publications.

Busko, D. A. (1998). Causes and consequences of perfectionism and procrastination: $A$ structural equation model. Tesis de maestría no publicada. Guelph, Ontario: University of Guelph.

Byrne, B. M. (2006). Structural equation modeling with EQS. Basic concepts, applications, and programming (2nd ed.). Hills Dale, NJ: Lawrence Erlbaum Associates.

Chau, C., \& Vilela, P. (2017). Determinantes de la salud mental en estudiantes universitarios de Lima y Huánuco. Revista de Psicología (PUCP), 35(2), 387-422.

Cid, P., Orellana, A., \& Barriga, O. (2010). Validación de la escala de autoeficacia general en Chile. Revista médica de Chile, 138(5), 551-557.

Cuadras, C. M. (2016). Problemas de probabilidades y estadística. (Vol. 2. Inferencia estadística). Barcelona: Edicions Universitat.

Damásio, B. F., Valentini, F., Núñes-Rodriguez, S. I., Kliem, S., Koller, S. H., Hinz, A., . . . Zenger, M. (2016). Is the general self-efficacy scale a reliable measure to be used in cross-cultural studies? Results from Brazil, Germany and Colombia. The Spanish journal of psychology, 19.

Domínguez Lara, S. A. (2014). ¿Matrices policóricas/tetracóricas o matrices Pearson? Un estudio metodológico. Revista Argentina de Ciencias del Comportamiento (RACC), $6(1), 39-48$.

Espada, J. P., Gonzálvez, M. T., Orgilés, M., Carballo, J. L., \& Piqueras, J. A. (2012). Validación de la Escala de Autoeficacia General con adolescentes españoles. Electronic Journal of Research in Educational Psychology, 10(26), 355-370.

Ferrando, P. J., \& Anguiano-Carrasco, C. (2010). El análisis factorial como técnica de investigación en psicología. Papeles del Psicólogo, 31(1), 18-33.

Flores, J., Mayorga-Vega, D., Blanco, R., \& Blanco, H. (2014). Perceived Self-Efficacy in Problem Solving and Scientific Communication in University Students. A Gender Study. Psychology, 5(5), 358-364. Doi: http://dx.doi.org/10.4236/psych.2014.55046.

Grimaldo Muchotrigo, M. (2005). Propiedades Psicométricas de la Escala de Autoeficacia General de Baessler \& Schwarzer. Revista Cultura, 19(1), 213-229.

Gutiérrez, L. R., Corrás, T., Novo, M., \& Fariña, F. (2017). El rendimiento académico: La influencia de las expectativas, el optimismo y la autoeficacia. Revista de Estudios $e$ Investigación en Psicología y Educación, Volumen Extra (10), 104-108.

IBM Corp. (2012). IBM SPSS Statistics for Windows. Armonk, NY: IBM Corp.

Jerusalem, M., \& Schwarzer, R. (1992). Self-efficacy as a resource factor in stress appraisal process. En R. Schwarzer (Ed.), Self-efficacy: Thought control of action (págs. 195211). Washington, DC: Hemisphere.

Kline, R. B. (2011). Principles and practice of structural equation modeling. New York, New York: Guilford Press.

Merino Tejedor, E., \& Lucas Mangas, S. (2016). La autoeficacia y la autorregulación como variables moderadoras del estrés laboral en docentes de educación primaria. Universitas Psychologica, 15(1), 205-218. 
Montero, I., \& León, O. (2007). A guide for naming research studies in Psychology. International Journal of Clinical and Health Psychology, 7(3), 847-862.

Moreta, R., Reyes, C., Mayorga, M., \& León-Tamayo, L. (2017). Estimación sobre niveles y factores de riesgo psicosocial en adolescentes escolarizados de Ambato, Ecuador. Pensando Psicología, 8(2), 29-40.

Moreta-Herrera, R., Duran-Rodriguez, T., \& Gaibor-Gonzalez, I. (2018). Factorial Structure and Realibility of Emotional Regulation Questionnaire (ERQ) in a Sample of Students From Ecuador. PSIENCIA-Revista Latinoamericana de Ciencia Psicológica, 10(2), Doi: 10.5872/psiencia/10.2.24.

Moreta-Herrera, R., Ilaja-Verdesoto, B., Mayorga-Lascano, M., León-Tamayo, L., \& LópezCastro, J. (2018). Actitudes y disposición personal ante el consumo y exposición a sustancias en adolescentes del Ecuador. Health \& Addictions/Salud y Drogas, 18(2), 217-226. Doi: 10.21134/haaj.v18i2.383.

Moreta-Herrera, R., López-Calle, C., Ramos-Ramírez, M., \& López-Castro, J. (2018). Estructura factorial y fiabilidad del Cuestionario de Salud General de Goldberg (GHQ12) en universitarios ecuatorianos. Revista Argentina de Ciencias del Comportamiento, 10(3), 35-42. Doi: 10.32348/1852.4206.v10.n3.20405.

Padilla, J. L., Acosta, B., Guevara, M., Gómez, J., \& González, A. (2006). Propiedades psicométricas de la versión española de la escala de autoeficacia general aplicada en México y España. Revista Mexicana de Psicología, 23(2), 245-252.

Peña Contreras, E. K., Lima Castro, S. E., Bueno Pacheco, G. A., Aguilar Sizer, M. E., Keyes, C. L., \& Arias Medina, W. P. (2017). Fiabilidad y validez de la Escala del Contínuum de Salud Mental (MHC-SF) en el contexto ecuatoriano. Ciencias Psicológicas, 11(2), 223-232.

Peter, C., Cieza, A., \& Geyh, S. (2014). Rasch analysis of the General Self-Efficacy Scale in spinal cord injury. Journal of health psychology, 19(4), 544-555.

Scholz, U., Doña, B. G., Sud, S., \& Schwarzer, R. (2002). Is general self-efficacy a universal construct? Psychometric findings from 25 countries. European Journal of Psychological Assessment, 18(3), 242-251. http://dx.doi.org/10.1027//1015-5759.18.3.242.

Schwarzer, R. (1993). Measurement of perceived self-eficacy. Psychomeiric scales for crosscirlrural research. Berlin, Germany: Freie Universitat Berlin.

Schwarzer, R., BaBler, J., Kwiatek, P., Schroder, K., \& Zhang, J. (1997). The Assessment of Optimistic Self-beliefs: Comparison of the German, Spanish, and Chinese Versions of the General Self-efficacy Scale. Applied Psychology, 46(1), 69-88.

Suárez, P. S., García, A. M., \& Moreno, J. B. (2000). Escala de autoeficacia general: datos psicométricos de la adaptación para población española. Psicothema, 12(Suplemento), 509-513.

Tabachnick, B. G., \& Fidell, L. S. (2007). Using multivariate statistics. Boston, Massachusetts: Pearson/Allyn \& Bacon. 